\title{
TGF- $\beta$ signaling controls FSHR signaling-reduced ovarian granulosa cell apoptosis through the SMAD4/miR-143 axis
}

\author{
Xing Du ${ }^{1}$, Lifan Zhang ${ }^{1}$, Xinyu $\mathrm{Li}^{1}$, Zengxiang Pan ${ }^{1}$, Honglin Liu ${ }^{1}$ and Qifa Li ${ }^{\star, 1}$
}

Follicle-stimulating hormone receptor (FSHR) and its intracellular signaling control mammalian follicular development and female infertility. Our previous study showed that FSHR is downregulated during follicular atresia of porcine ovaries. However, its role and regulation in follicular atresia remain unclear. Here, we showed that FSHR knockdown induced porcine granulosa cell (pGC) apoptosis and follicular atresia, and attenuated the levels of intracellular signaling molecules such as PKA, AKT and p-AKT. FSHR was identified as a target of miR-143, a microRNA that was upregulated during porcine follicular atresia. miR-143 enhanced pGC apoptosis by targeting FSHR, and reduced the levels of intracellular signaling molecules. SMAD4, the final molecule in transforming growth factor (TGF) $\beta$ signaling, bound to the promoter and induced significant downregulation of miR-143 in vitro and in vivo. Activated TGF- $\beta$ signaling rescued miR-143-reduced FSHR and intracellular signaling molecules, and miR-143-induced pGC apoptosis. Overall, our findings offer evidence to explain how TGF- $\beta$ signaling influences and FSHR signaling for regulation of pGC apoptosis and follicular atresia by a specific microRNA, miR-143.

Cell Death and Disease (2016) 7, e2476; doi:10.1038/cddis.2016.379; published online 24 November 2016

Follicle-stimulating hormone receptor (FSHR), a member of the rhodopsin-like $G$ protein-coupled receptor family, is specifically expressed on granulosa cells (GCs) of ovaries and Sertoli cells of testes. In ovaries of mammals, FSHR interacts with its ligand follicle-stimulating hormone (FSH) and has a crucial role in follicular development, steroidogenesis, and female infertility. ${ }^{1-4}$ In pigs, Sato et al. ${ }^{5,6}$ detected a quantitative trait loci (QTL) for the number of corpora lutea on SSC3 with genome-wide significance in an F2 resource population by crossing a Duroc boar with a Meishan sow, and further identified FSHR as a functional gene for this QTL by fine mapping, association, and mutational analyses. Maturation-dependent regulation of FSHR expression occurs in adult porcine ovaries. ${ }^{7}$ FSHR expression is downregulated during follicular atresia of pig ovaries, which is positively correlated with expression of CYP19A1, encoding a key aromatase enzyme in estradiol production, but is negatively correlated with progesterone levels in follicular fluid. ${ }^{8}$ However, the molecular mechanisms involved in the downregulation of FSHR during porcine follicular atresia are unclear.

Transforming growth factor (TGF)- $\beta$ signaling has a key role in life processes by complexing with its own membrane serine/ threonine kinase receptors TGFBR2 (type II receptor) and TGFBR1 (type I receptor), activating SMAD2/3 intracellular signaling, binding to SMAD4 in the nucleus, and then regulating transcription by inducing binding to target promoter regions termed SMAD-binding elements (SBEs). Activated TGF- $\beta$ signaling decreases porcine GC (pGC) apoptosis, whereas inactivated TGF- $\beta$ signaling enhances $\mathrm{pGC}$ apoptosis, indicating that TGF- $\beta$ signaling is required for $\mathrm{pGC}$ function. ${ }^{9,10}$ However, the mechanisms by which TGF- $\beta$ signaling regulates $\mathrm{pGC}$ apoptosis remain unexplored. In ovarian GCs of other mammals, such as humans, ${ }^{11}$ mice, ${ }^{12}$ rats, ${ }^{13}$ and cows, ${ }^{14}$ TGF- $\beta$ signaling is involved in FSHR expression and FSHR-induced cell function. Consistent with previous reports in mammals, TGF- $\beta$ signaling cooperates with FOXL2 to regulate FSHR expression and GC function in pre-hierarchical follicles of hens. ${ }^{15}$

Small RNAs (sRNAs) are non-coding RNA molecules of 21-24 nucleotides that have a vital role in multiple cellular physiological processes by silencing target genes at the posttranscriptional level. ${ }^{16,17}$ As an important type of sRNAs, microRNAs (miRNAs) are closely involved in mammalian ovarian GC functions such as GC apoptosis, ${ }^{18,19}$ proliferation, $^{20-22}$ and the cell cycle. ${ }^{22} \mathrm{Xu}$ et al. ${ }^{23}$ demonstrated the regulatory effect of miRNAs on pGC function for the first time. Thereafter, a number of studies focused on the role of miRNAs in pGCs. However, only a few miRNAs have been found to be associated with $p G C$ functions, such as miR-378, ${ }^{23,24}$ miR-26b, ${ }^{9,19,25}$ Let-7g, ${ }^{10,26}$ and miR-92a. ${ }^{27}$ Therefore, miRNA-mediated GC functions in pig ovaries need to be explored further.

In this study, we aimed to identify the miRNAs that target FSHR and to investigate the mechanism by which miRNAs regulate $\mathrm{pGC}$ function by targeting FSHR. Furthermore, we aimed to determine whether TGF- $\beta$ signaling is involved in the regulation of FSHR expression in pGCs, as has been shown in other mammals, ${ }^{11,14}$ and the effect of miRNAs targeting FSHR in this process.

\footnotetext{
${ }^{1}$ College of Animal Science and Technology, Department of Animal Genetics, Breeding and Reproduction, Nanjing Agricultural University, Nanjing, China *Corresponding author: Q Li, College of Animal Science and Technology, Nanjing Agricultural University, Weigang 1, Nanjing 210095, China. Tel: +86 25 8439 5046; Fax: +86 25 84395314; E-mail: liqifa@ njau.edu.cn 


\section{Results}

Involvement of FSHR in PGC apoptosis and follicular atresia. We previously demonstrated that FSHR is downregulated during porcine follicular atresia. ${ }^{8}$ To further investigate its role in follicular atresia, three FSHR-targeting small interfering RNAs (siRNAs) (siRNA-1, siRNA-2, and siRNA-3) were designed and individually transfected into pGCs. siRNA-3 had the highest knockdown efficiency at both the mRNA and protein levels in pGCs (Figures 1a and b) and was therefore used in the following experiments. Fluorescenceactivated cell sorting (FACS) analysis showed that knockdown of FSHR significantly increased the apoptosis rate of pGCs (Figure 1c), implying that knockdown of FSHR can induce pGC apoptosis in vitro. Next, we investigated the effect of FSHR knockdown on intracellular signaling in pGCs, and showed that the protein levels of protein kinase $A$ (PKA), AKT and p-AKT (Figure 1d) as well as the p-AKT/AKT ratio (Figure 1e) were sharply decreased, whereas expression of extracellular signal-regulated kinase 1/2 (ERK1/2) and p-ERK1/2 was not changed. In addition, silencing of FSHR attenuated $\mathrm{FSH}$-induced FSHR expression (Figure 1f), $\mathrm{FSH}$ reduced pGC apoptosis (Figure $1 \mathrm{~g}$ ), and $\mathrm{FSH}$-induced intracellular signaling (Figures $1 \mathrm{~h}-\mathrm{i}$ ). These results show that FSHR knockdown induces pGC apoptosis and follicular atresia, and its intracellular signaling molecules (PKA, AKT, and $\mathrm{p}-\mathrm{AKT}$ ) are possibly involved in this process.

FSHR is a novel target of miR-143. To explore the mechanism underlying differential expression of FSHR during porcine follicular atresia, four algorithms were used to predict the candidate miRNAs targeting FSHR. Only one miRNA (miR-143) targeting FSHR was commonly predicted by all four algorithms (Figure 2a). A putative miR-143-binding site was detected at the 286-293 nt position of the porcine FSHR $3^{\prime}$ untranslated region (3'UTR) (Figure 2b). BLAST analysis showed that the mature sequence of pig miR-143 is highly consistent with that in other vertebrates (Figure $2 \mathrm{c}$ ), and the seed sequence is complementary with the FSHR 3'UTR in pigs and other mammals (Figure 2d). To investigate whether miR-143 targets FSHR, we constructed a dual-luciferase reporter vector containing the wild-type miR-143-binding site located in the 3'UTR of FSHR or a mutated version (Figure 2e) and co-transfected it together with miR-143 mimics into pGCs. Luciferase activity analysis showed that miR-143 overexpression significantly decreased the luciferase activity of the wild-type reporter (Figure 2f), but had no effect on that of the mutated reporter (Figure $2 \mathrm{~g}$ ). These results suggest that FSHR is a direct target of miR-143.

miR-143 suppresses FSHR expression in pGCs. We next evaluated the effect of miR-143 on FSHR expression in pGCs. Quantitative RT-PCR (qRT-PCR) and western blot analyses showed that miR-143 overexpression significantly decreased the FSHR mRNA level (Figure 3a) and protein level (Figure 3b), suggesting that miR-143 can inhibit FSHR expression in pGCs. To confirm this, a specific inhibitor of miR-143 was designed and transfected into pGCs. The miR-143 level was markedly reduced by the miR-143 inhibitor (Figure 3c). Moreover, knockdown of miR-143 significantly increased the FSHR mRNA level (Figure 3d) and protein level (Figure $3 e$ ). We also detected the protein levels of FSHR intracellular signaling molecules (PKA, AKT, and p-AKT) and showed that overexpression of miR-143 decreased their expression levels (Figure $3 f$ ) and the $\mathrm{p}$-AKT/AKT ratio (Figure $3 \mathrm{~g}$ ), whereas knockdown of miR-143 significantly increased their expression levels in pGCs (Figure $3 \mathrm{~h}$ ) and the p-AKT/AKT ratio (Figure 3i). Taking these results together, we proved that miR-143 inhibits FSHR and its intracellular signaling molecules in pGCs.

miR-143 modulates pGC apoptosis and follicular atresia by targeting FSHR. Our previous study showed that miR-143 is upregulated during follicular atresia using a miRNA microarray assay. ${ }^{25}$ qRT-PCR demonstrated that the miR-143 level was higher in atretic follicles than in healthy follicles (Figure 4a). FACS analysis revealed that miR-143 overexpression significantly increased the pGC apoptosis rate (Figure 4b), whereas knockdown of miR-143 decreased the pGC apoptosis rate (Figure 4c), suggesting that miR-143 can promote pGC apoptosis. To determine whether FSHR mediates miR-143induced pGC apoptosis, pGCs were co-transfected with miR-143 mimics and FSHR-targeting siRNA. This showed that silencing of FSHR could increase miR-143-induced pGC apoptosis (Figure $4 d$ ). In addition, inhibition of miR-143 rescued FSHR-targeting siRNA-induced pGC apoptosis (Figure 4e). These results reveal that miR-143 regulates $p G C$ apoptosis and follicular atresia by targeting FSHR.

SMAD4 inhibits miR-143 expression by directly binding to its promoter. Two SMAD4-binding sites were found at positions $-359 /-356 \mathrm{nt}$ and $-306 /-303 \mathrm{nt}$ in the promoter of the miR-143 gene (Figure 5a), indicating that the transcription factor SMAD4 regulates miR-143 expression. To clarify this, we investigated the effect of SMAD4 on miR-143 expression in pGCs. Overexpression of SMAD4 significantly decreased miR-143 levels (Figure 5b), whereas knockdown of SMAD4 significantly increased expression of miR-143 (Figure $5 \mathrm{c}$ ). Furthermore, luciferase reporter vectors containing the wildtype or mutated SBE were constructed (Figure 5d) and transfected with pcDNA3.1-SMAD4 into pGCs. Luciferase activity analysis found that overexpression of SMAD4 decreased the luciferase activity of the wild-type SBE and SBE2-mut constructs, but had no effect on the SBE1-mut construct, suggesting that SMAD4 inhibits miR-143 promoter activity by binding to SBE1 in its promoter (Figure 5e). A chromatin immunoprecipitation (ChIP) assay confirmed that SMAD4 bound to $-359 /-356 \mathrm{nt}$ in the promoter of miR-143 (Figure 5f). All these results reveal that SMAD4 regulates miR-143 expression by directly binding to its promoter in vitro and in vivo.

SMAD4 positively regulates FSHR expression by targeting miR-143. We further examined the effect of SMAD4 on the miR-143 target FSHR in pGCs. Western blotting revealed that the FSHR protein level was significantly upregulated by SMAD4 overexpression (Figure 6a) and downregulated by knockdown of SMAD4 (Figure 6b), indicating that SMAD4 positively regulates FHSR expression in pGCs. To evaluate whether miR-143 mediates this process, 
a

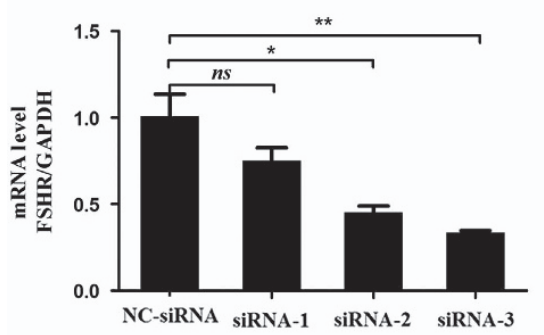

d

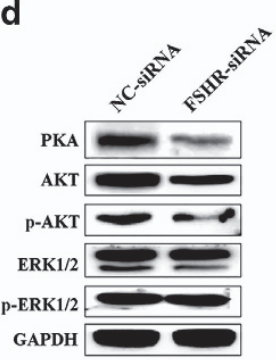

b

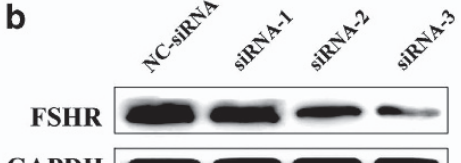

GAPDH

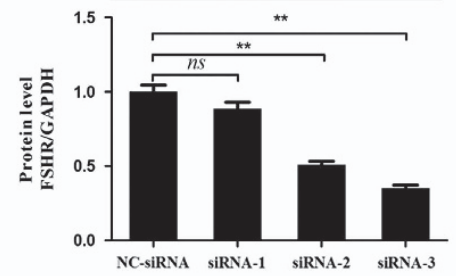

C

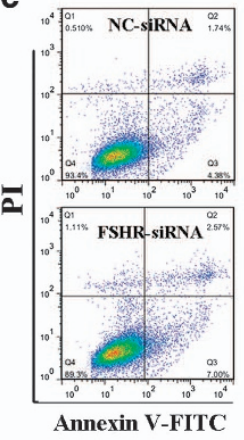

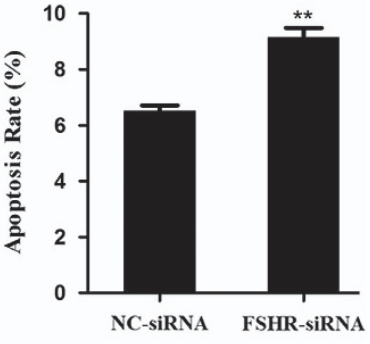

$f$ FSHR $-\cdots$
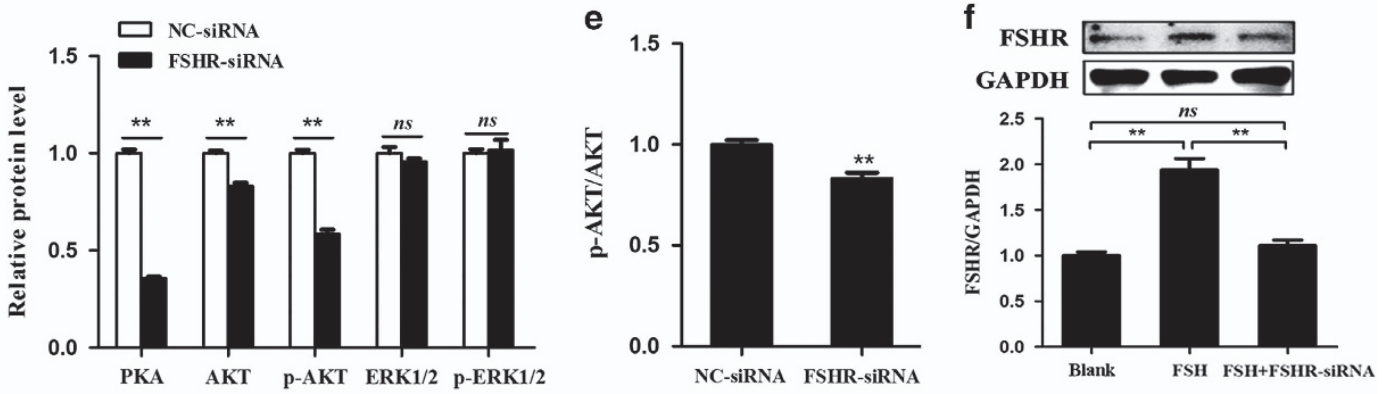

g
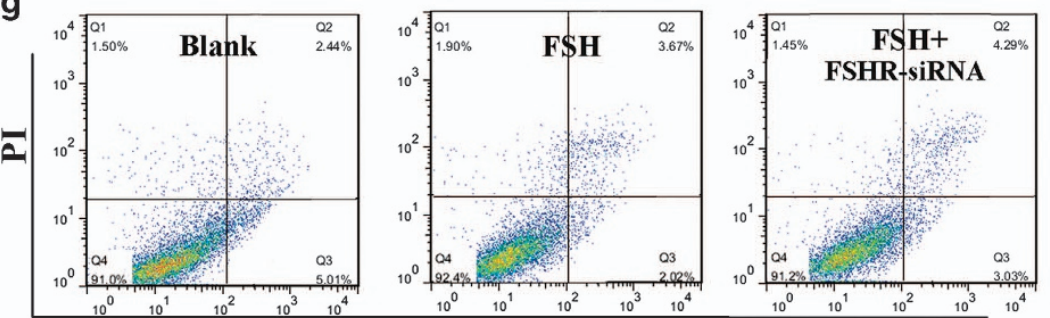

Annexin V-FITC

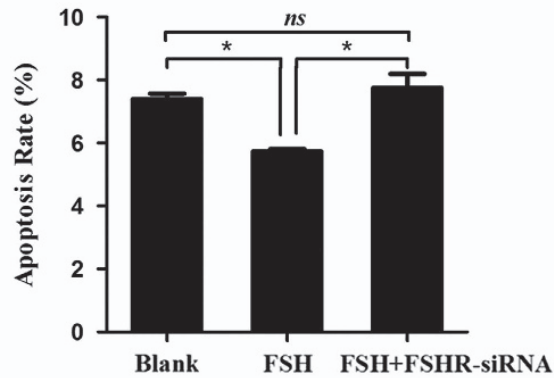

h

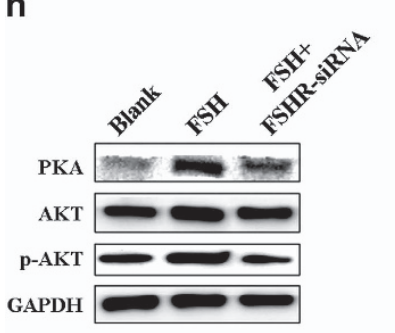

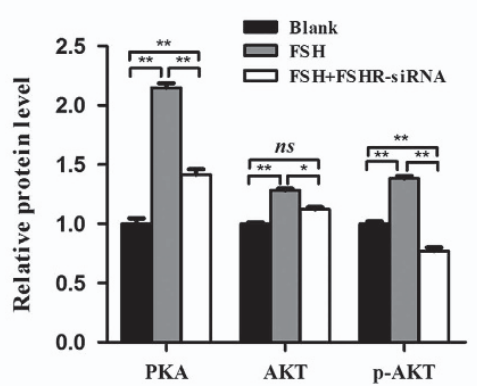

i

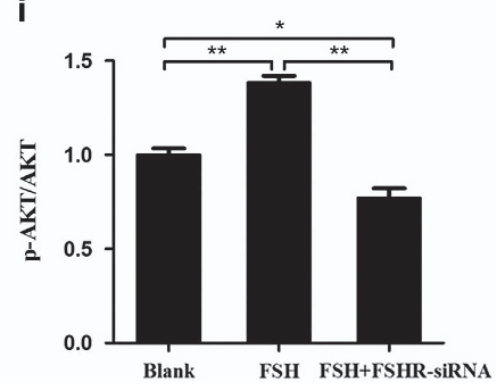

Figure 1 Knockdown of FSHR increases the apoptosis rate of pGCs. (a and $\mathbf{b}$ ) Silencing of FSHR expression in pGCs using siRNAs. qRT-PCR (a) and western blot (b) analyses showed that FSHR-targeting siRNAs (siRNA-2 and siRNA-3) provided optimal depletion of FSHR in pGCs compared with negative control (NC)-siRNA. (c) Knockdown of FSHR increases the apoptosis rate of pGCs. pGCs transfected with NC-siRNA or FSHR-targeting siRNA were subjected to Annexin V-FITC/PI double staining and flow cytometric analysis. (d) The effect of FSHR knockdown on its intracellular signaling levels. (e) The change in the p-AKT/AKT ratio in pGCs transfected with FSHR-targeting siRNA. (f-i) Knockdown of FSHR inhibits FSH-mediated function. After pGCs were co-treated with FSH (6.0 IU/ml) and FSHR-targeting siRNA, western blotting was performed to measure the levels of FSHR (f) and intracellular signaling molecules ( $h$ and i), and flow cytometry was performed to analyze the apoptosis rate of $p G C s(g)$. Average results from three independent experiments are shown. ${ }^{*} P<0.05,{ }^{* *} P<0.01$

we co-transfected pcDNA3.1-SMAD4 and miR-143 mimics. miR-143 significantly inhibited SMAD4-induced FSHR expression (Figure 6c). In addition, miR-143 significantly decreased SMAD4-induced expression of FSHR intracellular signaling molecules (PKA, AKT, and p-AKT) (Figure 6d) and the $\mathrm{p}$-AKT/AKT ratio (Figure $6 \mathrm{e}$ ). All the results are in line with the notion that SMAD4 positively regulates FSHR expression by targeting miR-143 in pGCs.

TGF- $\beta$ signaling regulates the miR-143/FSHR axis in pGCs. SMAD4 is the final core member of the TGF- $\beta$ signaling pathway. To determine whether SMAD4 
a

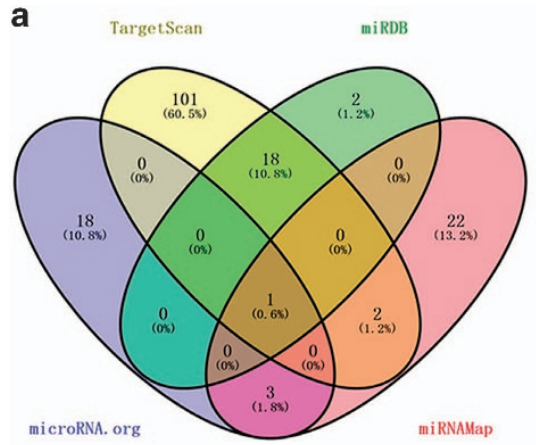

b

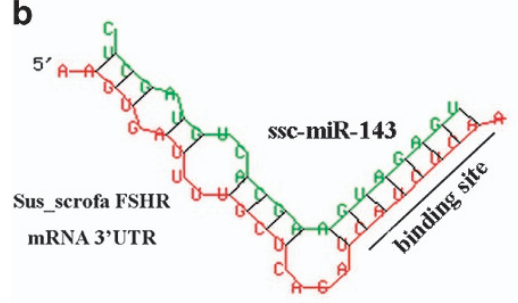

$\mathrm{mfe}:-22.1 \mathrm{kcal} / \mathrm{mol}$

\section{C}

$$
43 \text { Seed sequence }
$$

43 UGAGAUGAAGCACUGUAGCUC

hsa-miR-143 UGAGAUGAAGCACUGUAGCUC

mmu-miR-143 UGAGAUGAAGCACUGUAGCUC

rno-miR-143 UGAGAUGAAGCACUGUAGCUC

oar-miR-143 UGAGAUGAAGCACUGUAGCUC

pma-miR-143 UGAGAUGAAGCACUGUAACUCC

aca-miR-143 UGAGAUGAAGCACUGUAGCUC

ggo-miR-143 UGAGAUGAAGCACUGUAGCUCA

ptr-miR-143 UGAGAUGAAGCACUGUAGCUCA

Ppy-miR-143 UGAGAUGAAGCACUGUAGCUCA

Cfa-miR-143 UGAGAUGAAGCACUGUAGCUC

bta-miR-143 UGAGAUGAAGCACUGUAGCUCG

dre-miR-143

UGAGAUGAAGCACUGUAGCUC

*********************

d

Seed squence
miR-143 3'-CUCGAUGUCACGA

pig FSHR-3'UTR TTCAAAGTGATTTTGCTCAGATCATCTCAACTGA

human FSHR-3'UTR TTCAAAGTGCTCTTTGTCAGATCATCTCAACTGA

sheep FSHR-3'UTR ITCAAAGTGCTCTTGTTCAGATCATCTCAACTGA

$* * * * * * * * * * * * * * * * * * * * * * * * * * * * * * *$

e

3'-UTR

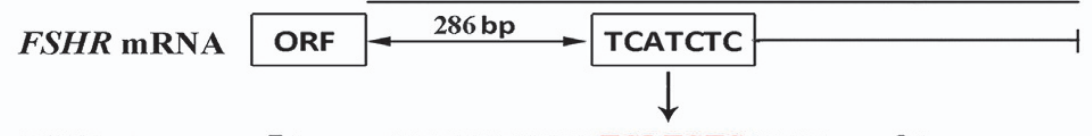

FSHR-wt $\quad 5^{\prime}-\ldots$ TTTGCTCAGATCATCTCAACT . . 3'

miR-143 3'-CUCGAUGUCACGAAGUAGAGU-5'

FSHR-mut $\quad 5^{\prime}-\ldots$ TTTGCTCAGAGTGCAGAAACT. . . ${ }^{\prime}$

f

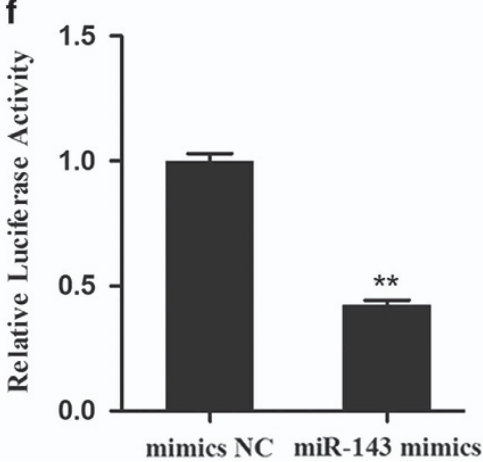

g

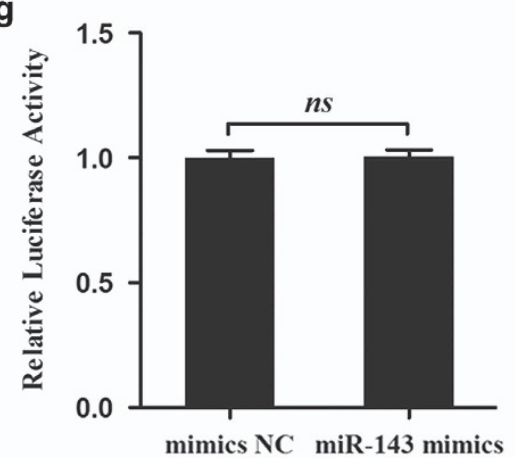

Figure 2 FSHR is a direct target of miR-143. (a) The candidate miRNAs that target FSHR were predicted by four different algorithms (TargetScan, miRDB, microRNA.org, and miRNAMap). miR-143 is the only miRNA to be predicted by all the algorithms, as shown in the Venn diagram. (b) The miR-143-binding site in the 3 'UTR of porcine FSHR and the minimal free energy (mfe) were predicted by RNAhybrid. (c) miR-143 mature sequences are highly conserved among vertebrates. Nucleotides that diverge from the human sequence are highlighted in red. Asterisks indicate complementarity. ssc, Sus scrofa; hsa, Homo sapiens; mmu, Mus musculus; rno, Rattus norvegicus; oar, Ovis aries; pma, Petromyzon marinus; aca, Anolis carolinensis; ggo, Gorilla gorilla; ptr, Pan troglodytes; ppy, Pongo pygmaeus; cfa, Canis familiaris; bta, Bos taurus; dre, Danio rerio. (d) Alignments of miR-143 mature sequences and the 3'UTR of FSHR from pig, human, and sheep. Red letters indicate the seed sequences of miR-143. Asterisks indicate complementarity. (e) Schematic showing the interactions of miR-143 with wild-type FSHR (red) and the mutant version (blue). (f and g) Transfection of pGCs with miR-143 mimics and a dual-luciferase reporter vector containing the wild-type FSHR 3'UTR (f) or the mutant version (g). Luciferase activity was measured and normalized to that in the mimics NC group. Experiments were conducted in triplicate. ${ }^{* *} P<0.01$

downstream of the miR-143/FSHR axis is regulated by TGF- $\beta$ signaling, pGCs was treated with different concentrations of TGF- $\beta 1(0,10$, and $20 \mathrm{ng} / \mathrm{ml})$ to activate TGF- $\beta$ signaling. qRT-PCR analysis showed that the miR-143 level was suppressed sharply by TGF- $\beta 1$ in a dose-dependent manner
(Figure 7a). The mRNA levels of FSHR and SMAD4 were significantly elevated in TGF- $\beta 1$-treated pGCs (Figure 7b). Similarly, the FSHR protein level was significantly increased in pGCs treated with TGF- $\beta 1$ (Figure 7c). In addition, miR-143 inhibited TGF- $\beta 1$-induced FSHR expression in 
a

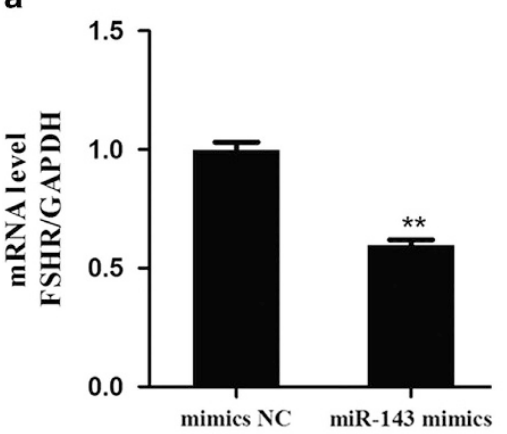

d

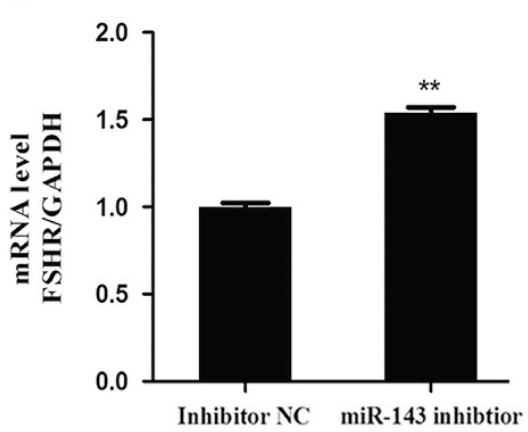

b

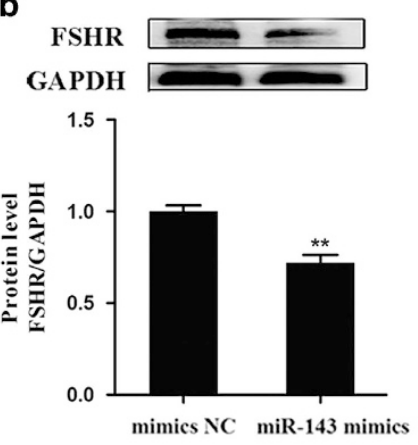

e

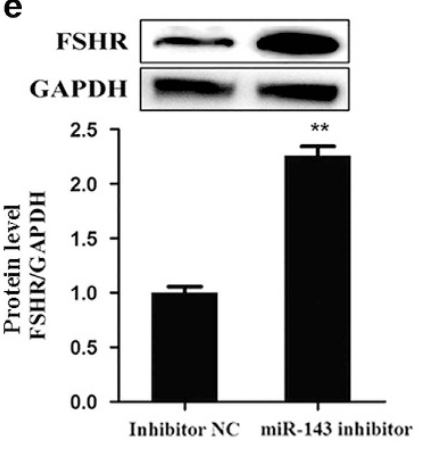

C

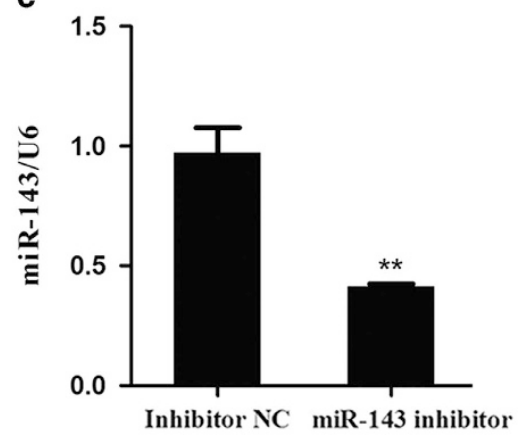

f
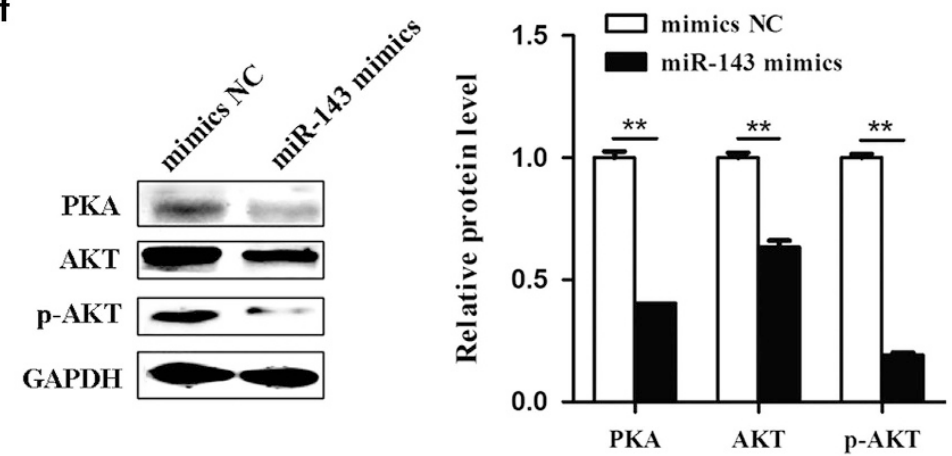

h
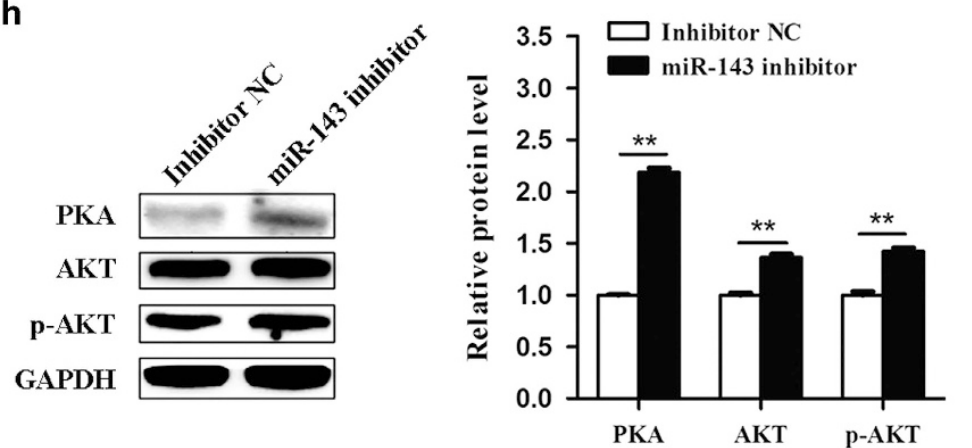

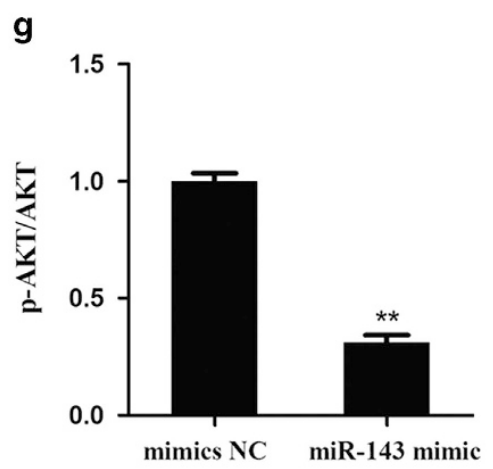

i

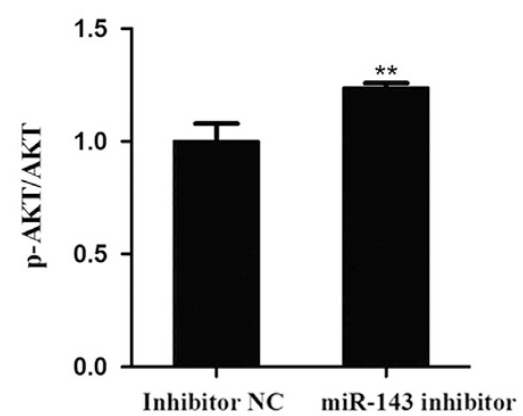

Figure 3 Effects of miR-143 on the expression of FSHR in pGCs. (a and $\mathbf{b}$ ) Mimics NC or miR-143 mimics were transfected into pGCs. mRNA levels of FSHR (a) were detected by qRT-PCR analysis and protein levels (b) were detected by western blotting. (c-e) A specific inhibitor of miR-143 was transfected into pGCs, and miR-143 levels (c) were detected by miRNA qRT-PCR with normalization to that of U6. FSHR mRNA (d) and protein (e) levels were measured, and GAPDH was used as an internal control. (f and $\mathbf{g}$ ) Overexpression of miR-143 regulates the intracellular signaling levels of FSHR (f) and the p-AKT/AKT ratio $(\mathbf{g})$ in pGCs. ( $h$ and i) Silencing of miR-143 regulates the intracellular signaling levels of FSHR (h) and the $\mathrm{p}-\mathrm{AKT} / \mathrm{AKT}$ ratio (i) in pGCs. ${ }^{* \star} P<0.01$ 
a

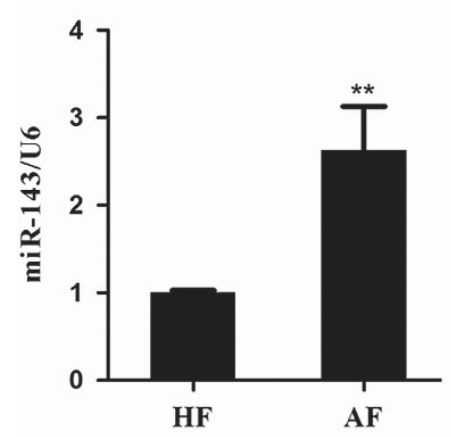

C

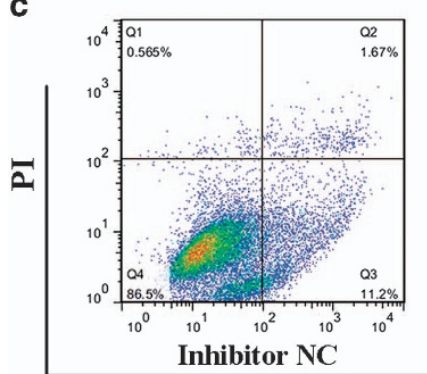

b
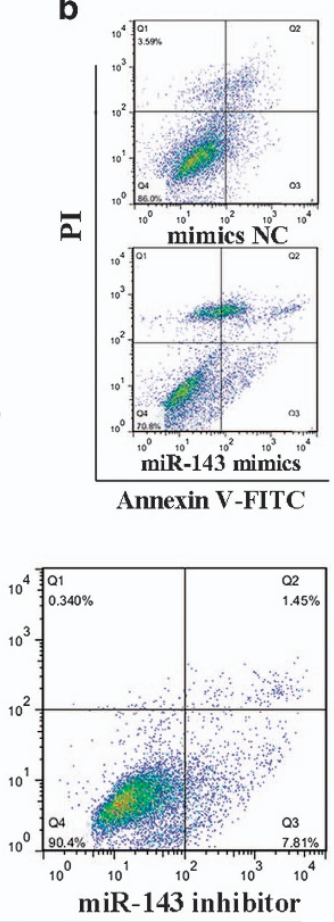

miR-143 inhibitor

Annexin V-FITC

d
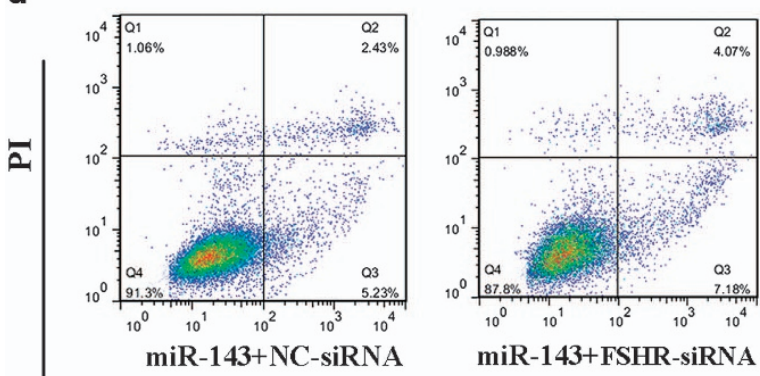

Annexin V-FITC

e

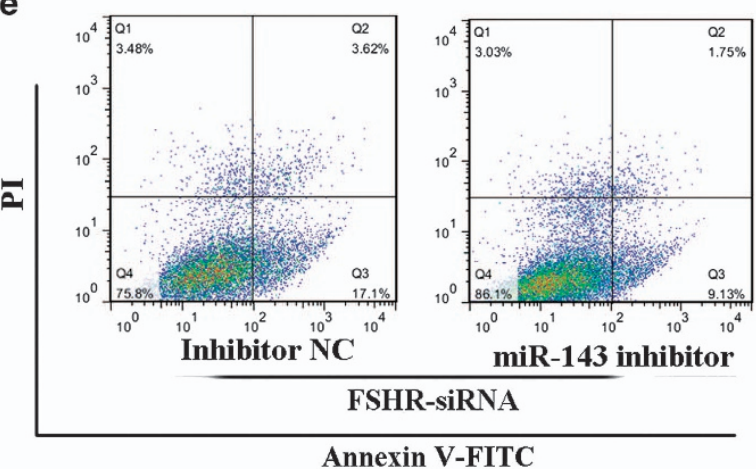

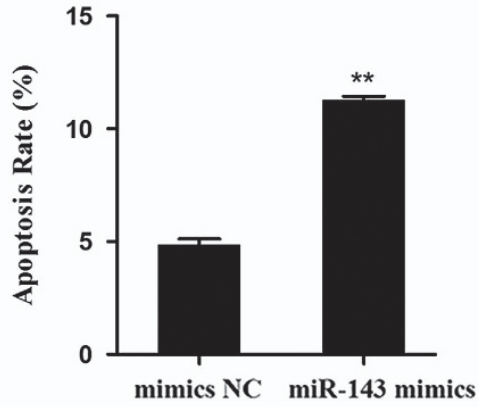
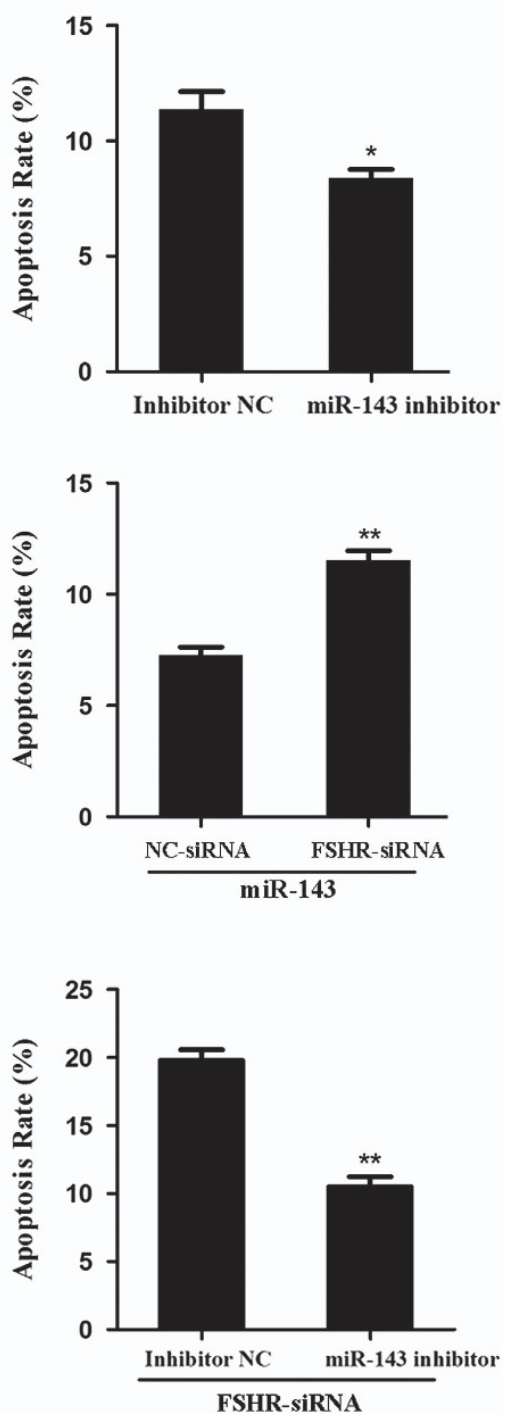

Figure 4 miR-143 induces pGC apoptosis by suppressing FSHR. (a) The expression levels of miR-143 were detected in healthy follicles (HF, $n=6)$ and atretic follicles $(A F, n=6)$. ( $(\mathbf{b}$ and $\mathbf{c})$ The apoptosis rate was detected in pGCs transfected with miR-143 mimics (b) or a miR-143 inhibitor (c). (d) miR-143 mimics were co-transfected with NCsiRNA or FSHR-targeting siRNA into pGCs, and the apoptosis rate was calculated by Annexin V-FITC/PI staining and FACS. (e) FSHR-targeting siRNA was co-transfected with a miR-143 inhibitor or an inhibitor NC into pGCs, and the apoptosis rate was calculated. ${ }^{*} P<0.05,{ }^{\star \star} P<0.01$ 

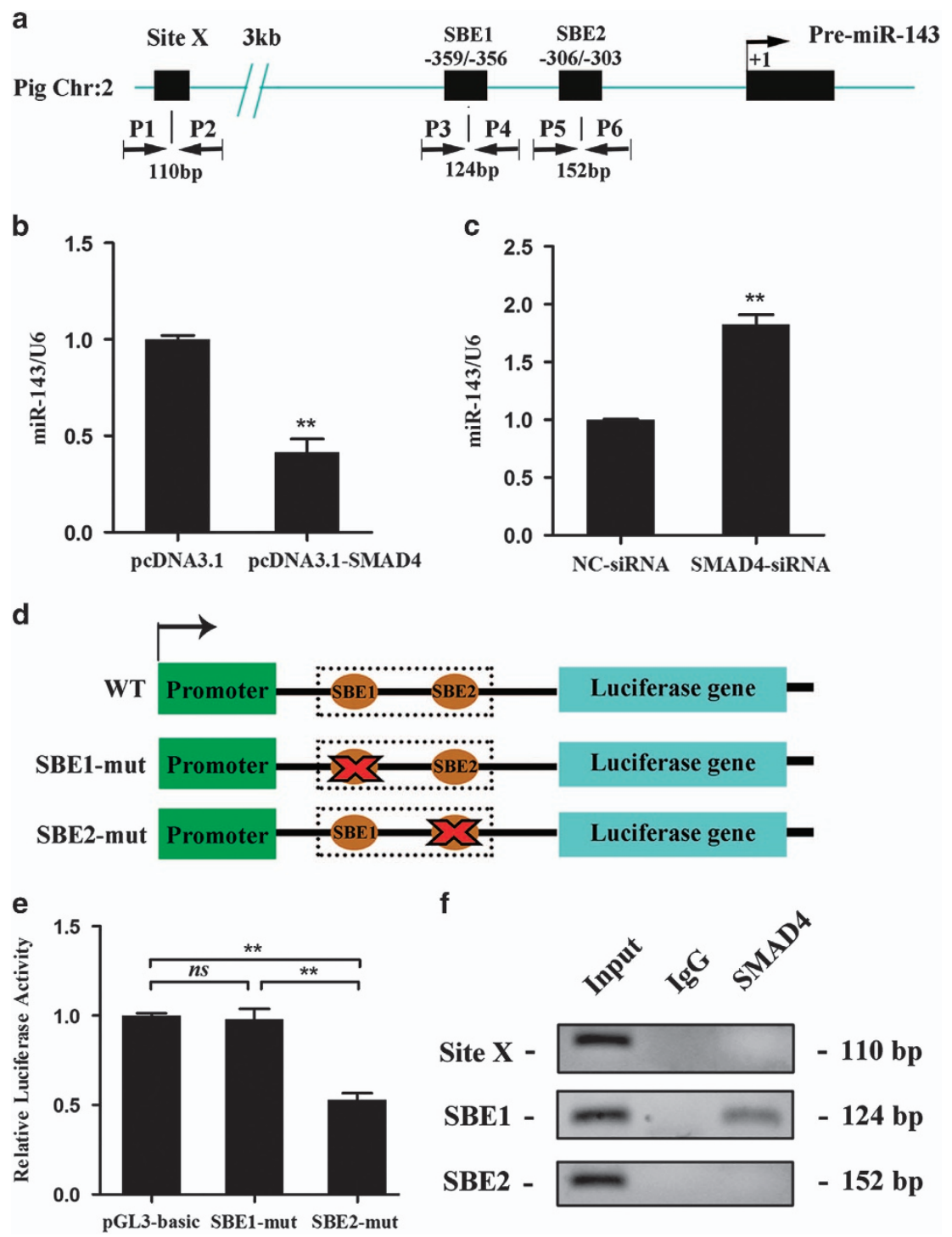

Figure 5 SMAD4 inhibits miR-143 expression in pGCs by binding to its promoter in vitro and in vivo. (a) A schematic diagram of the miR-143 promoter region. P1-P6 are the primers used for the ChIP assay. Nucleotide numbering is relative to +1 at the transcriptional start site. SBE, SMAD4-binding sites. Sites $X$, negative control locus for the ChIP assay. ( $b$ and c) SMAD4 regulates miR-143 expression in pGCs. SMAD4 was overexpressed (b) or knocked down (c) in pGCs and miR-143 levels were measured, with U6 as the endogenous control. (d) The luciferase reporter vector of the pig miR-143 promoter. The miR-143 promoter with the wild-type or mutated SBE was cloned upstream of the luciferase gene. Blue box, luciferase gene; green box, pGL-3 promoter; dotted box, miR-143 promoter; brown ovals, SBE1 and SBE2; red forks, mutation; WT, wild-type. (e) Luciferase activity was analyzed after pcDNA3.1-SMAD4 was co-transfected with the luciferase reporter vector. Data are normalized to the pGL-3-basic vector. (f) The ChIPqPCR assay was performed to identify the interaction between SMAD4 and the miR-143 promoter. IgG was used as a negative control. Site X was used as a negative control locus. Input, total RNA was reverse-transcribed before incubation with the anti-SMAD4 antibody and amplified with primers (P1-P6). Triplicate samples were analyzed for each treatment, and the results are presented as the mean \pm S.E.M. ${ }^{*} P<0.05$, ${ }^{\star \star} P<0.01$

pGCs (Figure 7c) and TGF- $\beta 1$ reduced pGC apoptosis (Figure $7 \mathrm{~d}$ ). These results showed that TGF- $\beta$ signaling controls miR-143/FSHR axis-induced apoptosis in pGCs.

\section{Discussion}

In mammalian ovaries, the $\mathrm{FSH}$-specific transmembrane receptor FSHR is specifically located on GCs, is a common marker gene of GCs, and is an important modulator of GC function. Activation of FSHR signaling strengthens GC proliferation and function, ${ }^{11,18,28}$ whereas silencing of FSHR signaling weakens GC proliferation and function. ${ }^{29,30}$ Our previous study showed that FSHR is downregulated during porcine follicular atresia. ${ }^{8}$ In this study, we further demonstrated that FSHR can control pGC apoptosis and follicular atresia. Moreover, silencing of FSHR decreased the levels of its intracellular signaling molecules such as PKA, ${ }^{11} \mathrm{AKT},{ }^{30}$ and $\mathrm{p}-\mathrm{AKT}$ in pGCs. In GCs, binding of FSH leads to the activation of FSHR, which can activate intracellular signaling. A recent report demonstrated that enhancement of the FSHR/ PKA signaling pathways should promote pGC proliferation. ${ }^{28}$ These results suggested that silencing of FSHR enhances 

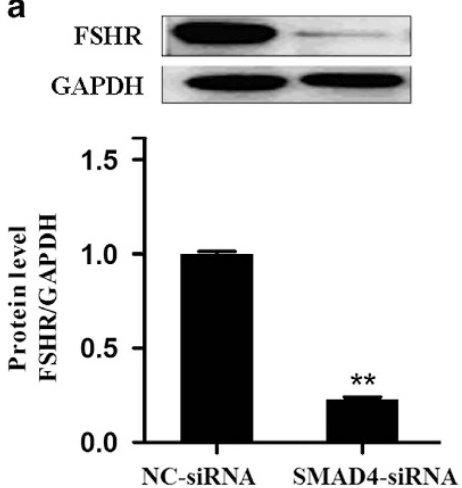

b
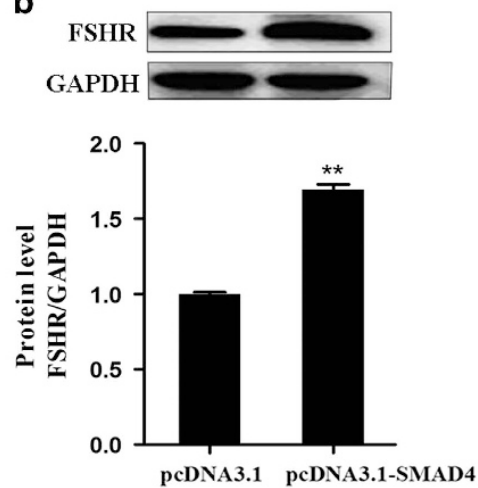

C
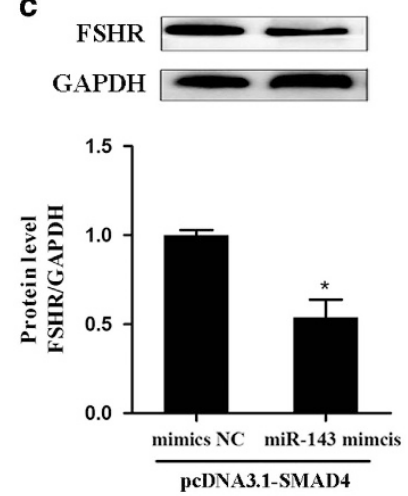

d

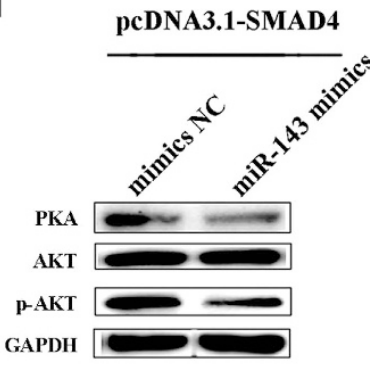

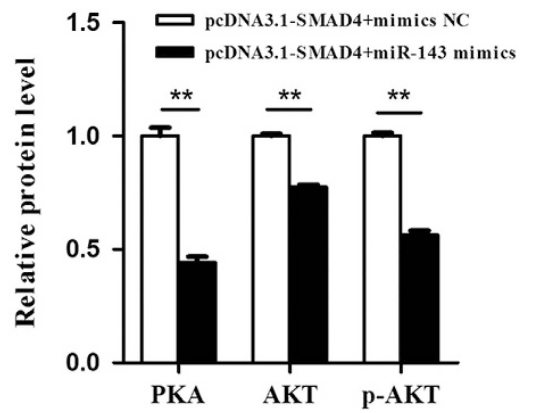

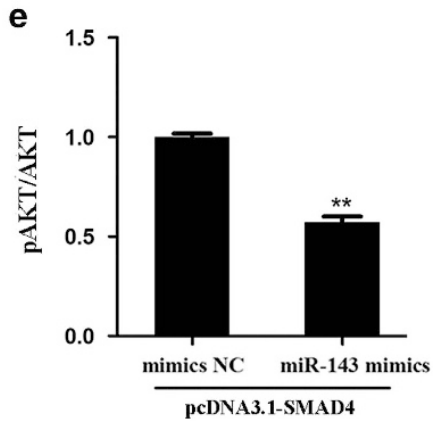

Figure 6 SMAD4 regulates FSHR expression via miR-143 in pGCs. (a and $\mathbf{b})$ SMAD4 regulates the FSHR protein level in pGCs. Western blot analyses were conducted to detect the FSHR protein level in pGCs transfected with SMAD4-targeting siRNA (a) or pcDNA3.1-SMAD4 (b). (c-e) miR-143 mediates SMAD4 regulation of FSHR and intracellular signaling. miR-143 mimics were co-transfected with pcDNA3.1-SMAD4 into pGCs. The protein levels of FSHR (c), PKA, AKT, and p-AKT (d) were measured by western blotting, and the $\mathrm{p}-\mathrm{AKT} / \mathrm{AKT}$ ratio (e) was calculated. ${ }^{*} P<0.05,{ }^{* *} P<0.01$

apoptosis, and attenuates the levels of its intracellular signaling molecules, such as PKA, AKT, and p-AKT.

As a key gonadotropin hormone receptor and a potential target for the treatment of fertility disorders, regulation of FSHR transcription has been extensively analyzed, especially its 5 ' regulatory region. ${ }^{31-33}$ A number of putative binding sites for transcription factors were detected in the 5 ' regulatory region of mammalian FSHR genes. Some of these transcription factors, such as GATA-1, ${ }^{34} \mathrm{E} 2 \mathrm{~F}^{34} \mathrm{SF}-1,{ }^{35}$ and USF-1, ${ }^{36}$ regulate $\mathrm{FSHR}$ expression by binding to its $5^{\prime}$ regulatory region. In addition, metastasis-associated protein 2, a component of histone deacetylase, can recruit histone deacetylase-1 to the FSHR promoter and participates in the downregulation of FSHR expression upon FSH treatment. ${ }^{31}$ In pGCs, we recently showed that histone H3K9 acetylation regulates expression of the FSHR gene. ${ }^{33}$ However, transcriptional regulation of its $3^{\prime} U T R$ remains unclear. In this study, we identified miR-143 as a novel miRNA targeting FSHR and demonstrated that miR-143 inhibits FSHR expression and function in pGCs by directly binding to its 3 'UTR. This result strongly suggested that the $3^{\prime} U T R$ has an essential role in the regulation of FSHR transcription.

miR-143 is an intronic miRNA, is a member of the miR-143/145 cluster, and is co-transcribed from the same gene as miR-145. ${ }^{37}$ Accumulating evidence shows that miR-143 is involved in the regulation of various cellular processes, such as normal cell apoptosis, proliferation, differentiation, migration, and self-renewal, ${ }^{38-40}$ as well as cancer cell apoptosis, proliferation, invasion, and autophagy. ${ }^{41-44}$ Notably, miR-143 has been extensively studied in cell apoptosis. Some apoptosis-related genes have been identified as miR-143 targets in multiple cell types, such as BCL-2 in gastric cancer cells ${ }^{45}$ and osteosarcoma cells, ${ }^{44}$ BAG3 (BCL-2-associated athanogene 3) in glioblastoma stem

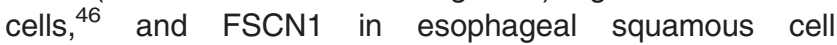
carcinoma. ${ }^{47}$ However, the role of miR-143 in GC apoptosis remains unknown. In this study, we demonstrated that $\mathrm{miR}-143$ is a pro-apoptotic factor and induces pGC apoptosis and follicular atresia.

As an important signaling pathway in the body, TGF- $\beta$ signaling may have a core role via crosstalk with other signaling pathways such as the FSHR signaling pathway. ${ }^{11}$ In this study, we showed that TGF- $\beta$ signaling enhanced FSHR expression and its intracellular signaling, consistent with previous reports in other mammals. ${ }^{11,12,48}$ Addition of TGF- $\beta$ increased FSHR mRNA expression in a time-dependent manner in rat GCs, ${ }^{48}$ whereas TGF- $\beta 1$ in the presence of FSH decreased FSHR expression in bovine GCs. ${ }^{14}$ SMAD3, a core component of TGF- $\beta$ signaling, is a signaling intermediate for TGF- $\beta$. Activated SMAD3-mediated signaling can increase expression of FSHR and its intracellular signaling, ${ }^{11,13}$ and also potentiates the ovarian response to FSH stimulation in GCs. ${ }^{12}$ These results indicated that TGF- $\beta$ signaling is involved in FSHR expression and FSHR-reduced pGC apoptosis. 
a

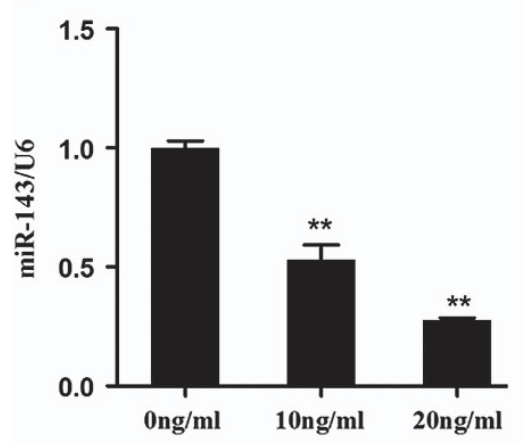

b

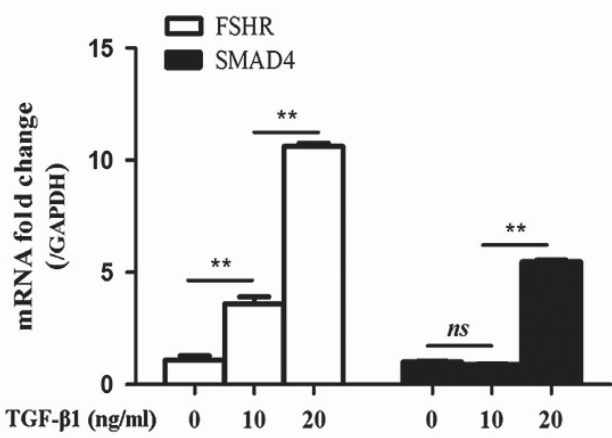

c

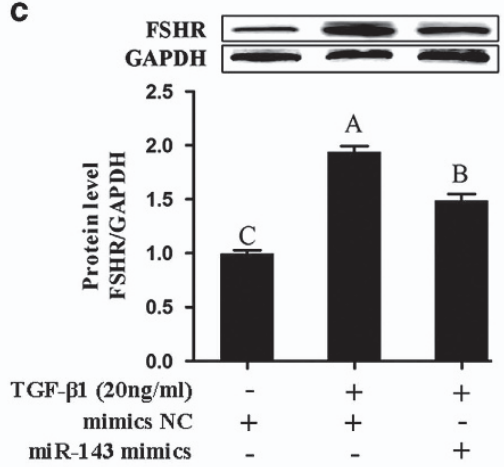

d
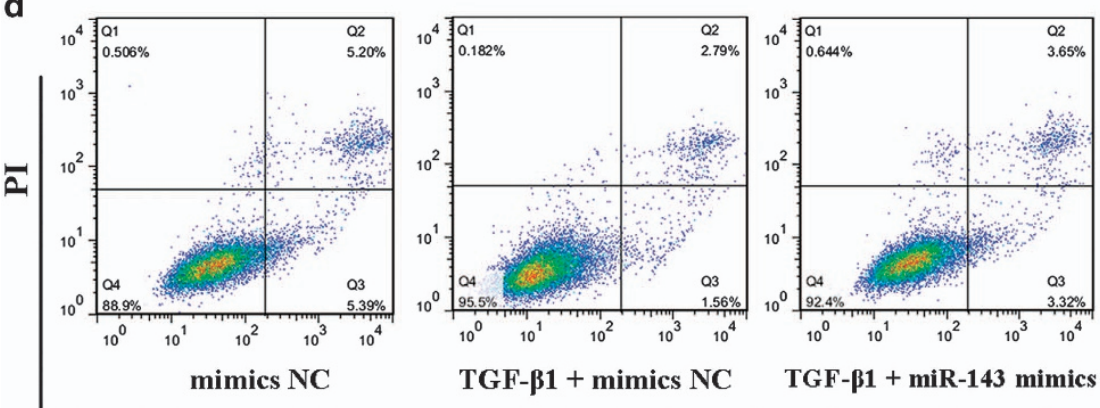

Annexin V-FITC

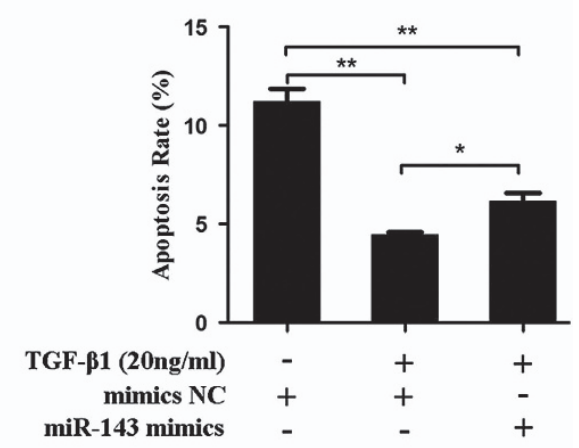

Figure 7 Effects of TGF- $\beta 1$ on the expression of miR-143 and FSHR. (a) TGF- $\beta 1$ suppresses the expression of miR-143. qRT-PCR analysis was performed to determine the expression of miR-143 when pGCs were treated with different concentrations of TGF- $\beta 1(0,10$, and $20 \mathrm{ng} / \mathrm{ml})$. (b) The SMAD4 and FSHR expression levels in pGCs are increased by TGF- $\beta 1$ in a dose-dependent manner. (c) miR-143 reduces TGF- $\beta 1$-induced expression of FSHR. pGCs were treated with $20 \mathrm{ng} / \mathrm{ml}$ TGF- $\beta 1$ and mimics NC or miR-143 mimics, and then western blotting was performed. (d) miR-143 increases TGF- $\beta 1$ reduced apoptosis of pGCs. pGCs were treated with $20 \mathrm{ng} / \mathrm{ml}$ TGF- $\beta 1$ and mimics NC or miR-143 mimics, followed by Annexin V-FITC/PI double staining. ${ }^{*} P<0.05$; ${ }^{\star \star} P<0.01$

SMAD4, as an important transcription factor as well as the final core molecule in TGF- $\beta$ signaling, has a pivotal role in TGF- $\beta$ signal transduction. ${ }^{49,50}$ SMAD4 can complete extracellular TGF- $\beta$ signal transmission by combining with SBEs of target promoters. ${ }^{51,52}$ TGF- $\beta$ signaling has an important role during epithelial-mesenchymal transition. Specifically, binding of SMAD4 to the SBE within the promoter region of the CAR gene in breast epithelial cells ${ }^{50}$ and the $\mathrm{CDH} 2$ gene in human pancreatic ductal epithelium ${ }^{53}$ and non-small cell lung cancer cells ${ }^{51}$ is necessary for TGF- $\beta$-stimulated transcription. Activation of hepatic stellate cells (HSCs) by TGF- $\beta 1$ initiates hepatitis $B$ virus-associated fibrogenesis. It has been recently reported that the TGF- $\beta 1$-activated SMAD4 complex may upregulate CD147 expression by directly interacting with a specific SBE in the CD147 promoter, thereby controlling HSC migration. ${ }^{52}$ Here, we identified two SBEs in the miR-143 promoter and demonstrated that SMAD4 decreased miR-143 expression in pGCs by binding to SBE2 in its promoter. Interestingly, a recent study demonstrated that blockade of TGF- $\beta$ signaling decreases the miR-143 level in smooth muscle cells. ${ }^{38}$ These data demonstrated that SMAD4 inactivates transcription by directly interacting with the miR-143 promoter and mediates TGF- $\beta$ signaling-regulated FSHR expression together with miR-143. Meanwhile, our data also established the link between TGF- $\beta$ signaling and FSHR in GCs of mammalian ovaries (Figure 8).
In conclusion, our data provide direct evidence that FSHR is an anti-apoptotic factor in pGCs and its expression and intracellular signaling are decreased by miR-143. Meanwhile, the miR-143/FSHR axis, which controls pGC function, is regulated by TGF- $\beta$ signaling. A potential mechanism underlying regulation of the miR-143/FSHR axis by TGF- $\beta$ signaling is that SMAD4, a central component of the TGF- $\beta$ signaling pathway, directly binds to the SBE in the miR-143 promoter. These findings provide novel insights into the mechanism underlying GC apoptosis, follicular atresia, and follicular development in ovaries of mammals including humans.

\section{Materials and Methods}

Reagents. Porcine TGF- $\beta 1$ was purchased from R\&D Systems (Minneapolis, MN, USA). Antibodies against PKA, ERK1/2, and p-ERK1/2 were obtained from Sangon Biotech (Shanghai, China), those against AKT and p-AKT were from Cell Signaling Technology (Beverly, MA, USA), and those against FSHR and GAPDH as well as anti-rabbit and anti-mouse IgG were from Santa Cruz Biotechnology (Santa Cruz, CA, USA). DMEM-F-12, fetal bovine serum (FBS), and phosphate-buffered saline were obtained from Life Technologies (Carlsbad, CA, USA). Lipofectamine 2000 was purchased from Invitrogen (Carlsbad, CA, USA). Protease and phosphatase inhibitors were obtained from Roche (Basel, Switzerland). miR-143 mimics, miR-143 inhibitors, and siRNAs were all synthesized by GenePharma (Shanghai, China) (Supplementary Table S1).

Cell culture. Fresh porcine ovaries were obtained and transported back to the laboratory within $1 \mathrm{~h}$. pGC collection and pGC and $293 \mathrm{~T}$ cell culture were performed as previously described. ${ }^{9}$ All animal experiments were approved by the Animal Ethics Committee at Nanjing Agricultural University, China. 
Normal pGCs

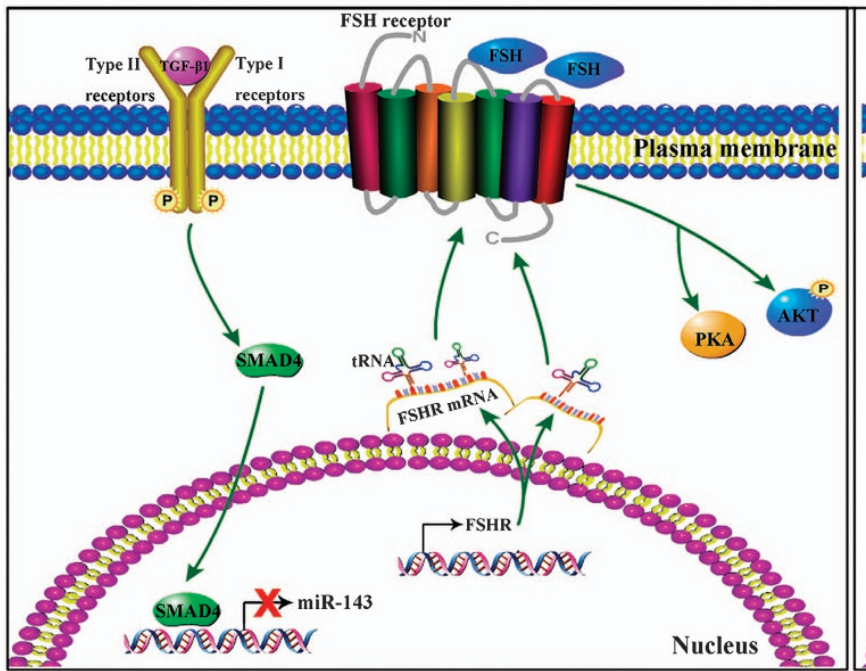

Apoptotic pGCs

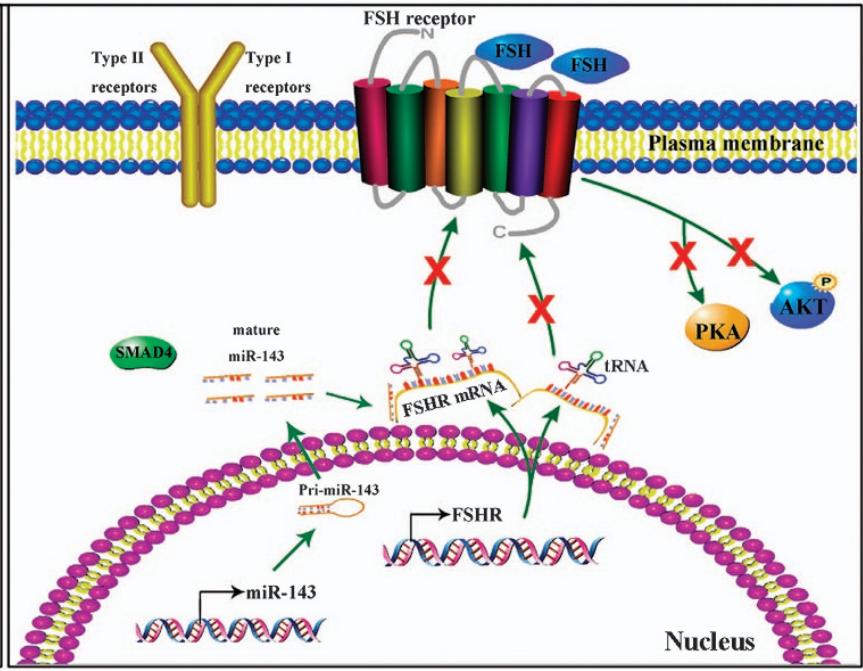

Figure 8 Model of crosstalk between TGF- $\beta$ signaling and FSHR signaling for regulation of pGC apoptosis

Cell treatments and transfection. pGCs were washed with phosphatebuffered saline and cultured in serum-free DMEM/F-12 containing $6.0 \mathrm{lU} / \mathrm{ml} \mathrm{FSH}$ (Ningbo Hormone Co., Ningbo, China) for $36 \mathrm{~h}$. For transfection, cells were seeded in six-well plates. After $12 \mathrm{~h}$, transfection was performed using Lipofectamine 2000 according to the manufacturer's protocol.

RNA isolation and qRT-PCR. Total RNA was isolated from pGCs using a High Purity Total RNA Extraction Kit (Bioteke, Beijing, China) and was then reversetranscribed into cDNA using PrimeScript ${ }^{\mathrm{TM}}$ RT Master Mix (TaKaRa, Dalian, China). qRT-PCR was performed using SYBR Green Master Mix (Vazyme Biotech, Nanjing, China) and relative expression levels were calculated using the $2^{-\Delta \Delta C t}$ method. The primers used are listed in Supplementary Table S2. The expression level of mature miR-143 was measured using a PrimeScript miRNA qPCR Starter Kit (TaKaRa). GAPDH and U6 small nuclear RNA were used as endogenous controls for genes and miRNAs, respectively.

Western blotting. Total protein was extracted from pGCs using radioimmunoprecipitation assay buffer containing $1 \%$ phosphatase inhibitor $(\mathrm{v} / \mathrm{V})$. The protein concentration was determined by the BCA method (Pierce, Shanghai, China). A $12 \%$ SDS-PAGE gel was prepared and $25 \mu \mathrm{g}$ of protein was loaded. After electrophoresis for $1 \mathrm{~h}$, the total protein was transferred to a PVDF membrane (Millipore, Billerica, MA, USA). The membrane was blocked in $5 \%$ non-fat milk and then incubated with primary antibodies overnight at $4{ }^{\circ} \mathrm{C}$. After washing, the appropriate secondary antibodies were used and chemiluminescence was detected.

Apoptosis analysis. The apoptosis rate of pGCs was measured using an Apoptosis Detection Kit (Vazyme Biotech) according to the manufacturer's protocol. In total, $2 \times 10^{5}$ cells were sorted by FACS using a cell counting machine (Becton Dickinson, Franklin, NJ, USA). The apoptosis rate was calculated using the following equation: (number of cells in the right upper quadrant+number of cells in the right lower quadrant)/(total number of cells).

Bioinformatics analysis. miRNAs that target FSHR were predicted by four online software programs, TargetScan (http://www.mirbase.org/), miRDB (http://www. mirdb.org/miRDB/), microRNA.org (http://www.microrna.org/), and miRNAMap (http://mirnamap.mbc.nctu.edu.tw/). RNAhybrid (http://bibiserv.techfak. uni-bielef eld.de/rnahybrid/) was used to predict the miR-143-binding sites in the $3^{\prime}$ UTR of FSHR. miR-143 mature sequences of different species were obtained from miRBase (http://www.mirbase.org/).

Plasmid construction. For miR-143-binding site detection, the pmirGLO Dual-luciferase miRNA Target Expression Vector containing the wild-type FSHR
3'UTR was constructed. For SMAD4-binding site detection, the pGL-3 reporter vector containing the wild-type miR-143 promoter was constructed. Mutant plasmids were constructed using a TaKaRa MutanBEST Kit (TaKaRa) according to the manufacturer's instructions. The pcDNA3.1-SMAD4 plasmid was prepared previously by our group. ${ }^{9}$

Luciferase reporter assay. After transfection for $24 \mathrm{~h}$, pGCs were collected and luciferase activities were measured using the Dual-Luciferase Reporter Assay System (Promega, Madison, WI, USA) according to the manufacturer's instructions.

Chromatin immunoprecipitation. In total, $1 \times 10^{7}$ pGCs were fixed in $1 \%$ formaldehyde and sonicated for $5 \mathrm{~min}$ (10-s on and 10-s off) on ice with a $3 \mathrm{~mm}$ microtip and the output control set to $30 \%$. The sonicated chromatin fluid $(800 \mu l)$ was collected by centrifugation. Then, sonicated chromatin $(400 \mu \mathrm{l})$ was diluted 2.5 -fold and mixed with protein $\mathrm{A} / \mathrm{G}$-agarose $(40 \mu \mathrm{l})$, followed by shaking at $4{ }^{\circ} \mathrm{C}$ for $1 \mathrm{~h}$. This mixture was then centrifuged at 5000 r.p.m. for $1 \mathrm{~min}$, and the supernatant was collected into a new dolphin tube. An anti-SMAD4 antibody and rabbit IgG $(2 \mu \mathrm{g})$ were added and the sample was incubated overnight at $4{ }^{\circ} \mathrm{C}$ with shaking. After crosslinking reversal and proteinase $\mathrm{K}$ treatment, DNA was released, precipitated, and diluted in 50-100 $\mu \mathrm{l}$ of $10 \mathrm{mM}$ Tris-HCL (pH 8.0). PCR-amplified products were analyzed on a $3 \%$ agarose gel.

TGF- $\boldsymbol{\beta} 1$ treatment. pGCs were seeded in six-well plates at a density of $1 \times 10^{6}$ cells per well and cultured in medium containing $10 \%$ FBS for $24 \mathrm{~h}$. Then, the medium was replaced with serum-free medium and TGF- $\beta 1$ was added to a final concentration of 10 and $20 \mathrm{ng} / \mathrm{ml}$. After $24 \mathrm{~h}$, cells were collected and the expression levels of miR-143, SMAD4, and FSHR were determined.

Statistical analysis. Statistical analysis was performed using IBM SPSS Statistics v20.0 (SPSS Inc., Chicago, IL, USA). An unpaired two-sided Student's $t$-test and a one-way analysis of variance were used to evaluate the significance of statistics. $P<0.05$ was considered statistically significant. All data are presented as means \pm S.E.M.

\section{Conflict of Interest}

The authors declare no conflict of interest.

Acknowledgements. This work was supported by the National Natural Science Foundation of China (no. 31572373, 31630072, and 31672421), the 
National Key Scientific Program (no. 2014CB138502), and the Jiangsu Agriculture Science and Technology Innovation Fund (no. SCX(12)2097).

1. Dias JA, Campo B, Weaver BA, Watts J, Kluetzman K, Thomas RM et al. Inhibition of follicle-stimulating hormone-induced preovulatory follicles in rats treated with a nonsteroidal negative allosteric modulator of follicle-stimulating hormone receptor. Biol Reprod 2014; 90: 19.

2. Plunkett J, Doniger S, Orabona G, Morgan T, Haataja R, Hallman M et al. An evolutionary genomic approach to identify genes involved in human birth timing. PLoS Genet 2011; 7: e1001365.

3. Ilgaz NS, Aydos OS, Karadag A, Taspinar M, Eryilmaz OG, Sunguroglu A. Impact of folliclestimulating hormone receptor variants in female infertility. J Assist Reprod Genet 2015; 32 : 1659-1668.

4. Bramble MS, Goldstein EH, Lipson A, Ngun T, Eskin A, Gosschalk JE et al. A novel folliclestimulating hormone receptor mutation causing primary ovarian failure: a fertility application of whole exome sequencing. Hum Reprod 2016; 31: 905-914.

5. Sato S, Atsuji K, Saito N, Okitsu M, Komatsuda A, Mitsuhashi T et al. Identification of quantitative trait loci affecting corpora lutea and number of teats in a Meishan x Duroc F2 resource population. J Anim Sci 2006; 84: 2895-2901.

6. Sato S, Hayashi T, Kobayashi E. Fine mapping the number of corpora lutea quantitative trait Ioci on SSC3: analysis of the porcine follicle-stimulating hormone receptor gene. Anim Sci J 2011; 82: 633-641.

7. Liu J, Aronow BJ, Witte DP, Pope WF, La Barbera AR. Cyclic and maturationdependent regulation of follicle-stimulating hormone receptor and luteinizing hormone receptor messenger ribonucleic acid expression in the porcine ovary. Biol Reprod 1998; 58 : 648-658.

8. Pan Z, Zhang J, Lin F, Ma X, Wang X, Liu H. Expression profiles of key candidate genes involved in steroidogenesis during follicular atresia in the pig ovary. Mol Biol Rep 2012; 39: 10823-10832.

9. Liu J, Du X, Zhou J, Pan Z, Liu H, Li Q. MicroRNA-26b functions as a proapoptotic factor in porcine follicular granulosa cells by targeting Sma-and Mad-related protein 4. Biol Reprod 2014; 91: 146.

10. Zhou J, Liu J, Pan Z, Du X, Li X, Ma B et al. The let-7 g microRNA promotes follicular granulosa cell apoptosis by targeting transforming growth factor- $\beta$ type 1 receptor. Mol Cell Endocrinol 2015; 409: 103-112.

11. Liu $Y$, Chen X, Xue X, Shen C, Shi C, Dong J et al. Effects of Smad3 on the proliferation and steroidogenesis in human ovarian luteinized granulosa cells. IUBMB Life 2014; 66: 424-437.

12. Gong $X, M c G e e$ EA. Smad3 is required for normal follicular follicle-stimulating hormone responsiveness in the mouse. Biol Reprod 2009; 81: 730-738.

13. Li Y, Jin Y, Liu Y, Shen C, Dong J, Xu J. SMAD3 regulates the diverse functions of rat granulosa cells relating to the FSHR/PKA signaling pathway. Reproduction 2013; 146: 169-179.

14. Zheng X, Price CA, Tremblay $Y$, Lussier JG, Carriere PD. Role of transforming growth factor- $\beta 1$ in gene expression and activity of estradiol and progesteronegenerating enzymes in FSH-stimulated bovine granulosa cells. Reproduction 2008; 136 : 447-457.

15. Qin N, Fan XC, Xu XX, Tyasi TL, Li SJ, Zhang YY et al. Cooperative effects of FOXL2 with the members of TGF- $\beta$ superfamily on FSH receptor mRNA expression and granulosa cell proliferation from hen prehierarchical follicles. PLoS One 2015; 10: e0141062.

16. Wei W, Zhang WY, Bai JB, Zhang HX, Zhao YY, Li XY et al. The NF-kappaB-modulated microRNAs miR-195 and miR-497 inhibit myoblast proliferation by targeting Igf1r, Insr and cyclin genes. J Cell Sci 2016; 129: 39-50.

17. Niu D, Lii YE, Chellappan P, Lei L, Peralta K, Jiang $C$ et al. miRNA863-3p sequentially targets negative immune regulator ARLPKs and positive regulator SERRATE upon bacterial infection. Nat Commun 2016; 7: 11324.

18. Sen A, Prizant H, Light A, Biswas A, Hayes E, Lee $H J$ et al. Androgens regulate ovarian follicular development by increasing follicle stimulating hormone receptor and microRNA-125b expression. Proc Natl Acad Sci USA 2014; 111: 3008-3013.

19. Liu J, Tu F, Yao W, Li X, Xie Z, Liu H et al. Conserved miR-26b enhances ovarian granulosa cell apoptosis through HAS2-HA-CD44-caspase-3 pathway by targeting HAS2. Sci Rep 2016; 6: 21197.

20. Yin M, Wang X, Yao G, Lu M, Liang M, Sun $Y$ et al. Transactivation of micrornA-320 by microRNA-383 regulates granulosa cell functions by targeting E2F1 and SF-1 proteins. J Biol Chem 2014; 289: 18239-18257.

21. Jiang L, Huang J, Li L, Chen $Y$, Chen $X$, Zhao $X$ et al. MicroRNA-93 promotes ovarian granulosa cells proliferation through targeting CDKN1A in polycystic ovarian syndrome. J Clin Endocrinol Metab 2015; 100: E729-E738.

22. Gebremedhn S, Salilew-Wondim D, Hoelker M, Rings F, Neuhoff C, Tholen E et al. MicroRNA-183 96 182 cluster regulate bovine granulosa cell proliferation and cell cycle transition by coordinately targeting FOXO1. Biol Reprod 2016; $94: 127$.

23. Xu S, Linher-Melville K, Yang BB, Wu D, Li J. Micro-RNA378 (miR-378) regulates ovarian estradiol production by targeting aromatase. Endocrinology 2011; 152: 3941-3951.
24. Toms D, Xu S, Pan B, Wu D, Li J. Progesterone receptor expression in granulosa cells is suppressed by microRNA-378-3p. Mol Cell Endocrinol 2015; 399: 95-102.

25. Lin F, Li R, Pan ZX, Zhou B, Yu de B, Wang XG et al. miR-26b promotes granulosa cell apoptosis by targeting ATM during follicular atresia in porcine ovary. PLOS One 2012; 7: e38640.

26. Cao R, Wu W, Zhou X, Liu K, Li B, Huang X et al. Let-7 $\mathrm{g}$ induces granulosa cell apoptosis by targeting MAP3K1 in the porcine ovary. Int $\mathrm{J}$ Biochem Cell Biol 2015; 68: 148-157.

27. Liu J, Yao W, Yao Y, Du X, Zhou J, Ma B et al. MiR-92a inhibits porcine ovarian granulosa cell apoptosis by targeting Smad7 gene. FEBS Lett 2014; 588: 4497-4503.

28. Cai L, Sun A, Li H, Tsinkgou A, Yu J, Ying S et al. Molecular mechanisms of enhancing porcine granulosa cell proliferation and function by treatment in vitro with anti-inhibin alpha subunit antibody. Reprod Biol Endocrinol 2015; 13: 26.

29. Chen MJ, Chou CH, Chen SU, Yang WS, Yang YS, Ho HN. The effect of androgens on ovarian follicle maturation: dihydrotestosterone suppress FSH-stimulated granulosa cell proliferation by upregulating PPARgamma-dependent PTEN expression. Sci Rep 2015; 5: 18319.

30. Wang XJ, Xiong GP, Luo XM, Huang SZ, Liu J, Huang XL et al. Dibutyl phthalate inhibits the effects of follicle-stimulating hormone on rat granulosa cells through down-regulation of follicle-stimulating hormone receptor. Biol Reprod 2016; 94 : 144.

31. Zhang S, Li W, Zhu C, Wang X, Li Z, Zhang J et al. Sertoli cell-specific expression of metastasis-associated protein 2 (MTA2) is required for transcriptional regulation of the follicle-stimulating hormone receptor (FSHR) gene during spermatogenesis. J Biol Chem 2012; 287: 40471-40483.

32. George JW, Dille EA, Heckert LL. Current concepts of follicle-stimulating hormone receptor gene regulation. Biol Reprod 2011; 84: 7-17.

33. Wu W, Han J, Cao R, Zhang J, Li B, Liu Z et al. Sequence and regulation of the porcine FSHR gene promoter. Anim Reprod Sci 2015; 154: 95-104.

34. Kim JS, Griswold MD. E2F and GATA-1 are required for the Sertoli cell-specific promoter activity of the follicle-stimulating hormone receptor gene. J Androl 2001; 22: 629-639.

35. Levallet J, Koskimies P, Rahman N, Huhtaniemi I. The promoter of murine follicle-stimulating hormone receptor: functional characterization and regulation by transcription factor steroidogenic factor 1. Mol Endocrinol 2001; 15: 80-92.

36. Viswanathan $\mathrm{P}$, Wood MA, Walker WH. Follicle-stimulating hormone (FSH) transiently blocks FSH receptor transcription by increasing inhibitor of deoxyribonucleic acid binding/ differentiation-2 and decreasing upstream stimulatory factor expression in rat Sertoli cells. Endocrinology 2009; 150: 3783-3791.

37. Kent OA, McCall MN, Cornish TC, Halushka MK. Lessons from miR-143/145: the importance of cell-type localization of miRNAs. Nucleic Acids Res 2014; 42: 7528-7538.

38. Climent M, Quintavalle M, Miragoli M, Chen J, Condorelli G, Elia L. TGFbeta triggers miR-143/145 transfer from smooth muscle cells to endothelial cells, thereby modulating vessel stabilization. Circ Res 2015; 116: 1753-1764.

39. Deng L, Blanco FJ, Stevens H, Lu R, Caudrillier A, McBride M et al. MicroRNA-143 activation regulates smooth muscle and endothelial cell crosstalk in pulmonary arterial hypertension. Circ Res 2015; 117: 870-883.

40. Gao Y, Han Z, Li Q, Wu Y, Shi X, Ai Z et al. Vitamin C induces a pluripotent state in mouse embryonic stem cells by modulating microRNA expression. FEBS J 2015; 282: 685-699.

41. Liu J, Mao Y, Zhang D, Hao S, Zhang Z, Li Z et al. MiR-143 inhibits tumor cell proliferation and invasion by targeting STAT3 in esophageal squamous cell carcinoma. Cancer Lett 2016; 373: 97-108.

42. Xu YF, Li YQ, Guo R, He QM, Ren XY, Tang XR et al. Identification of miR-143 as a tumour suppressor in nasopharyngeal carcinoma based on microRNA expression profiling. Int J Biochem Cell Biol 2015; 61: 120-128.

43. Du F, Feng Y, Fang J, Yang M. MicroRNA-143 enhances chemosensitivity of Quercetin through autophagy inhibition via target GABARAPL1 in gastric cancer cells. Biomed Pharmacother 2015; 74: 169-177.

44. Li WH, Wu HJ, Li YX, Pan HG, Meng T, Wang X. MicroRNA-143 promotes apoptosis of osteosarcoma cells by caspase-3 activation via targeting Bcl-2. Biomed Pharmacother 2016; 80: 8-15.

45. Zhuang M, Shi Q, Zhang X, Ding Y, Shan L, Shan X et al. Involvement of miR-143 in cisplatin resistance of gastric cancer cells via targeting IGF1R and BCL2. Tumour Biol 2015; 36: $2737-2745$.

46. Liu J, Qu CB, Xue YX, Li Z, Wang P, Liu YH. MiR-143 enhances the antitumor activity of shikonin by targeting BAG3 expression in human glioblastoma stem cells. Biochem Biophys Res Commun 2015; 468: 105-112.

47. Liu R, Liao J, Yang M, Sheng J, Yang H, Wang Y et al. The cluster of miR-143 and miR-145 affects the risk for esophageal squamous cell carcinoma through co-regulating fascin homolog 1. PLoS One 2012; 7: e33987.

48. Inoue K, Nakamura K, Abe K, Hirakawa T, Tsuchiya M, Oomori Y et al. Mechanisms of action of transforming growth factor beta on the expression of follicle-stimulating hormone receptor messenger ribonucleic acid levels in rat granulosa cells. Biol Reprod 2003; 69: $1238-1244$. 
49. Zhang L, Du X, Wei S, Li D, Li Q. A comprehensive transcriptomic view on the role of SMAD4 gene by RNAi-mediated knockdown. Reproduction 2016; 152: 81-89.

50. Vincent T, Neve EP, Johnson JR, Kukalev A, Rojo F, Albanell J et al. A SNAIL1-SMAD3/4 transcriptional repressor complex promotes TGF- $\beta$ mediated epithelial-mesenchymal transition. Nat Cell Biol 2009; 11: 943-950

51. Yang $\mathrm{H}$, Wang L, Zhao J, Chen Y, Lei Z, Liu X et al. TGF- $\beta$-activated SMAD3/4 complex transcriptionally upregulates $\mathrm{N}$-cadherin expression in non-small cell lung cancer. Lung Cancer 2015; 87: 249-257.

52. Li HY, Ju D, Zhang DW, Li H, Kong LM, Guo Y et al. Activation of TGF- $\beta 1-C D 147$ positive feedback loop in hepatic stellate cells promotes liver fibrosis. Sci Rep 2015; 5 16552.

53. Kang Y, Ling J, Suzuki R, Roife D, Chopin-Laly X, Truty MJ et al. SMAD4 regulates cell motility through transcription of $\mathrm{N}$-cadherin in human pancreatic ductal epithelium. PLOS One 2014; 9: e107948. (c) (i) Cell Death and Disease is an open-access journal published by Nature Publishing Group. This work is licensed under a Creative Commons Attribution 4.0 International License. The images or other third party material in this article are included in the article's Creative Commons license, unless indicated otherwise in the credit line; if the material is not included under the Creative Commons license, users will need to obtain permission from the license holder to reproduce the material. To view a copy of this license, visit http://creativecommons.org/licenses/by/4.0/

(C) The Author(s) 2016

Supplementary Information accompanies this paper on Cell Death and Disease website (http://www.nature.com/cddis) 